\title{
ARTICLE
}

\section{Why are long-acting injectable antipsychotics still underused?}

\author{
Peter Bosanac \& David Jonathan Castle
}

Peter Bosanac is Director of Clinical Services at St Vincent's Mental Health, Melbourne. His clinical work encompasses adult psychiatry, including acute inpatient, emergency and community psychiatry. He is an Associate Professor in the Department of Psychiatry at the University of Melbourne. His clinical research interests include eating disorders, acute psychiatry and obsessivecompulsive disorder (OCD). David Jonathan Castle is Chair of Psychiatry at St Vincent's Health and The University of Melbourne. He has broad clinical and research interests, encompassing schizophrenia and related disorders, bipolar disorder, cannabis misuse, OCD spectrum disorders and disorders of body image. Correspondence Professor David Jonathan Castle.

The University of Melbourne, Level 2-46 Nicholson Street, Fitzroy, Melbourne, Australia 3065. Email: david.castle@svha.org.au

\begin{abstract}
SUMMARY
'Depot antipsychotics' ('long-acting injectable antipsychotic medications' or LAls) are underused in the treatment of schizophrenia (including first episodes) and, possibly, of schizophrenia with comorbid substance use disorders. Patients' and clinicians' beliefs and attitudes, and service barriers, affect best practice and evidence-based care in LAI prescription. Poor medication adherence is a key reason for LAI prescription, but patients receiving LAls may still relapse or experience significant side-effects. Patients' and clinicians' attitudes towards antipsychotic medication, as well as the quality of their recovery-focused relationship, are key factors in adherence. Clinicians should avoid a dichotomous 'oral $v$. LAl' choice: LAls may have a place at various stages in the continuum of care and they should be one of the options discussed with any patient requiring long-term treatment, even early in the illness course. Many clinicians need better education about LAls and greater familiarity with schizophrenia treatment guidelines.
\end{abstract}

\section{LEARNING OBJECTIVES}

- Understand the contemporary benefits and risks of LAls in the continuum of care of schizophrenia

- Appreciate the barriers to the use of LAls, including stigma and clinicians' attitudes

- Understand the role of a recovery-focused approach to optimising therapeutic utility of LAls

\section{DECLARATION OF INTEREST}

P.B. has received grant monies for research and travel support from Astra Zeneca. D.C. has received grant monies for research, and/or travel support and honoraria for talks and consultancy from Allergen, Astra Zeneca, Bristol-Myers Squibb, Eli Lilly, Hospira, Janssen Cilag, Lundbeck, Organon, Pfizer, Roche, Sanofi, Servier and Wyeth; he is a current Advisory Board Member for: bitopertin (Roche); Lu AA21004, asenapine and aripiprazole LAI (Lundbeck); desvenlafaxine and varenicline (Pfizer); quetiapine (Astra Zeneca).

It has been a decade since Patel $\&$ David's review in Advances titled 'Why aren't depot antipsychotics prescribed more often and what can be done about it?' (Patel 2005). Increasingly, the term 'long-acting injectable antipsychotic medication' (LAI) has been adopted in preference to the more stigmatising 'depot' and the latter's association with limitations attributed to first-generation antipsychotics (Patel 2009). The burgeoning range of LAIs has largely been one of reformulations of existing medications, rather than the development of novel agents, and the use of LAIs has continued to fall in many jurisdictions (Patel 2010). Poor adherence to maintenance antipsychotics, rather than patient preference, is still the primary indication for LAIs (Patel 2009). The continuing challenges with LAIs include:

- adherence, even when suboptimal adherence is more overt than with oral agents

- variable prescribing by clinicians, with a focus mostly on long-standing rather than first-episode schizophrenia

- variable acceptance by patients and clinicians

- increasing concern regarding tolerability.

There is now greater emphasis on recoveryfocused care and supported decision-making, rather than overly paternalistic relationships between clinicians and patients (Chopra 2009). This collaborative shift in the therapeutic dyad encompasses shared decision-making about LAIs (Haddad 2011) and hence joint discussion and consideration of medication options, and their benefits $v$. risks, as well as adherence. Although the risk of extrapyramidal side-effects (e.g. tardive dyskinesia), particularly with (first-generation) early LAIs, has continued (Kane 2009), there is now a greater spotlight on cardiometabolic sequelae, including diabetes, obesity and hyperlipidaemia (de Hert 2009). Accordingly, we review the rate of utilisation of 'depot' or LAI medication in the context of the changing clinical landscape.

\section{Schizophrenia and adherence: are LAls part of the answer?}

Schizophrenia is the only widely researched indication for LAIs. The overall prevalence of suboptimal adherence among people with schizophrenia who are prescribed antipsychotic medication ranges from one- to two-thirds (Patel 
2009). Although there has been much early research and support for maintenance treatment with LAIs in patients with schizophrenia who exhibit poor medication adherence, use of this route in this clinical population in the USA has ranged from 19 to only 30\% (West 2008). In a national sample of US psychiatrists, LAI prescribing was inversely related to the prior use of second-generation oral antipsychotics and other oral psychotropic medications (West 2008). In the UK, about 30\% of patients with schizophrenia are prescribed LAIs (Barnes 2009). However, the long-term outcome in schizophrenia is heterogeneous, whether or not maintenance antipsychotic medication is prescribed (Harrow 2007). Although patients and clinicians alike often overestimate adherence (Velligan 2007), poor adherence with LAIs is more obvious than with oral medications.

\section{First-episode psychosis}

Irrespective of the route (oral or LAI), treatment adherence in people with schizophrenia is associated with fewer relapses and readmissions to in-patient care (Staring 2010; Offord 2013). Although patients with first-episode schizophrenia are the least likely to be prescribed LAIs, they are also at substantial risk of the consequences of suboptimal treatment or poor adherence, including the impact of relapse. Consequently, consideration of the role of LAIs has broadened from relapse prevention in people with longstanding schizophrenia and poor adherence, to include people with first-episode psychosis. It has been argued that this might facilitate earlier treatment that is more effective in the longer term, rather than waiting for a person to enter a chronic relapsing phase of illness that is only partially responsive to medication (Emsley 2008). Nevertheless, sufficient time to evaluate patients referred to early intervention services is required before either oral antipsychotics or LAIs are initiated, as a substantial proportion of referrals turn out not to have a first-episode psychotic disorder. Indeed, in one 4-year observational study, $41 \%$ of referrals to an early intervention service were 'non-cases': significantly, almost half had a major depressive or anxiety disorder (O’Donoghue 2012).

In addition to the ambivalence of treating clinicians about treatment recommendations, the limited use of LAIs in first-episode psychosis is likely compounded by the views of carers and families, including their attitudes and beliefs about mental illness, their level of acceptance of it, and whether any perceived risks outweigh benefits (Kane 2009).

\section{Schizophrenia and comorbid substance use}

Although LAIs have been prescribed to people with schizophrenia and comorbid substance use (Green 2007; Koola 2012), particularly when there are concerns about medication adherence, the supporting evidence for such use is limited and pertains to case reports and open-label studies of flupentixol, zuclopenthixol, risperidone (Koola 2012) and paliperidone (Vázquez Vázquez 2012). Two studies suggesting a favourable effect of LAI risperidone in this population (Rubio 2006; Rosenheck 2011) require replication and evaluation via randomised controlled trials (Green 2012).

\section{Recommendations in clinical guidelines}

Most clinical guidelines for the treatment of psychotic disorders - including those of the National Institute for Health and Care Excellence (NICE 2014), the American Psychiatric Association (Lehman 2010), the Canadian Psychiatric Association (Addington 2005), the Schizophrenia Patient Outcomes Research Team (PORT) (Kreyenbuhl 2010) and Texas Medication Algorithms (Argo 2007) - pay regard to patient preference and recommend, albeit vaguely, that LAIs be prescribed for patients with recurrent relapses linked to suboptimal adherence or non-adherence. However, these guidelines have not recommended that LAIs be used in patients with schizophrenia who favour oral medications and have been consistent in their adherence to these, nor in patients who have experienced significant poor tolerability or response to LAIs (Kane 2009).

\section{The evidence that LAls enhance adherence and outcomes}

\section{First-generation LAIs v. oral antipsychotics}

An observational study of patients who had been prescribed oral or LAI haloperidol or fluphenazine showed that those prescribed LAIs were twice as likely to remain in treatment and had a longer period until all-cause medication discontinuation (Zhu 2008). In patients with first-episode schizophrenia or schizoaffective disorder, there were lower rates of readmission to hospital and discontinuation of LAI perphenazine compared with oral haloperidol (Tiihonen 2006).

A synthesis of Cochrane systematic reviews of first-generation LAIs found a slight benefit on global functioning in comparison with oral antipsychotics, but there were no differences between individual LAIs (Adams 2001). These findings were probably limited by an underrepresentation of individuals with poor adherence to oral antipsychotics in randomised 
controlled studies (Adams 2001; Tiihonen 2011). A Cochrane review comparing zuclopenthixol decanoate with other first-generation LAIs in schizophrenia revealed superior efficacy in delaying or preventing relapse, but at the expense of more adverse side-effects (da Silva Freire Coutinho 2009).

A 3-year international observational study of first-generation LAIs compared with oral olanzapine favoured the latter (Haro 2007). However, a nationwide cohort study in Finland found that, compared with oral antipsychotics, LAIs (first generation and risperidone) were associated with reduced risk of readmission (Tiihonen 2011).

\section{First- v. second-generation LAls and oral antipsychotics}

A meta-analysis of first- and second-generation LAIs found benefits over oral agents in nonrandomised observational, but not in randomised, studies (Kirson 2013). Other systematic reviews of first- and second-generation LAIs compared with oral antipsychotics (Leucht 2011; Zhornitsky 2012) found them to be associated with a reduced rate of relapse. Also, a 2-year multicentre randomised study comparing risperidone LAI with equivalently dosed oral second-generation antipsychotics demonstrated reduced readmission rates, but no difference in terms of discontinuation of treatment (Rosenheck 2011).

A multicentre randomised controlled study comparing haloperidol with paliperidone LAI in patients with schizophrenia or schizoaffective disorder found 'efficacy failure' (defined as any of: increased out-patient visits, crisis stabilisation, in-patient admission or inability to discontinue oral antipsychotics within 8 weeks of LAI commencement) in a third of participants (McEvoy 2014). Although the study did not find haloperidol or paliperidone to differ in the rate of 'efficacy failure' over 2 years, haloperidol was associated with a greater rate of akathisia, and paliperidone with greater weight gain and elevated serum prolactin.

\section{Cost-effectiveness}

In general, second-generation LAIs are considerably more expensive than first-generation LAIs. Such considerations, which are particularly relevant to healthcare services, need to be balanced against the effectiveness, differential side-effect profile and tolerability of medications in individual patients, thus affording more personalised medicine. A systematic review of 28 economic evaluations of LAIs for schizophrenia found that second-generation LAIs, particularly risperidone, were likely to be a more cost-effective initial management strategy than haloperidol LAI and other LAI or oral formulations. However, the review was limited by utilisation of qualitative (not quantitative) assessment and the unavailability or exclusion of possibly relevant papers and conference abstracts (Achilla 2013).

\section{Acceptability of LAls}

Validated recovery approaches in psychiatry emphasise:

- the centrality of the patient's goals

- psychoeducation, cognitive-behavioural strategies to support treatment adherence, relapse prevention plans, and social and coping skills training

- the therapeutic relationship to identify strengths, resources and goals (Chopra 2009).

Consequently, patients' expectations and acceptance of LAIs need to be considered in the context of the quality of the patient-clinician relationship (McCabe 2012). Using a collaborative recovery approach when discussing the benefits and risks of LAIs with patients emphasises their sense of self, social inclusion and relationships, instilling hope rather than focusing mainly on symptoms and impairments. The compulsory administration of LAIs under mental health legislation because the patient is at risk of harming themselves or others does not mitigate the onus on the clinician to take a recovery-based approach: patients who perceive coercion in their contact with mental health services are less likely to be engaged in care (Stanhope 2009).

Not surprisingly, the acceptability of LAIs to people with schizophrenia is also influenced by their previous experience of them (Heres 2007), and side-effects adversely affect adherence (Dibonaventura 2012). A survey of patients' views about LAIs found that two-thirds were not given sufficient information by their clinicians. Concerns about losing autonomy and about pain secondary to injections fuelled negative views about this treatment (Jaeger 2010). However, the majority of patients who had actually received LAIs had positive attitudes towards them (Walburn 2001; Heres 2007; Waddell 2009), whereas only a minority of patients who had not tried them found the suggestion of LAIs acceptable (Heres 2007).

It seems that clinicians often view LAIs as a last-resort treatment to mitigate the risk of relapse (Waddell 2009). Prescribing practices vary not only within but also between countries. Clinicians in the USA in particular tend to hold 
negative attitudes towards LAIs (Patel 2009). A survey of US psychiatrists found that less than one-fifth of patients were prescribed LAIs for poor adherence to oral medications (West 2008). Among psychiatrists surveyed in the UK, 38\% thought that LAIs were less effective in patients with first-episode psychosis and a third thought that patients preferred oral medications (Patel 2010). Despite their view that LAIs were associated with superior adherence compared with oral medications, half of the surveyed psychiatrists had reduced their prescribing of LAIs in the preceding 5 years. A survey of psychiatrists, patients and relatives in Switzerland found that fewer than one in ten psychiatrists prescribed an LAI following a first episode of psychosis (Jaeger 2010). This study also found that patients were more negative about LAIs than their relatives or psychiatrists, mostly because of the above-mentioned concerns about their autonomy being restricted and experiencing pain as a result of the injections.

\section{Antipsychotic polypharmacy}

Antipsychotic polypharmacy is more likely in patients treated with LAIs (Barnes 2009) and in those with suboptimal response to a single LAI (Patel 2009). A study of patients in the USA found that they were less likely to be prescribed LAIs together with adjunctive oral antipsychotics than their counterparts in Europe (Olfson 2007). Polypharmacy may also contribute to clinicians' views about less favourable tolerability of LAIs compared with oral medications (Patel 2009).

\section{Prescribing guidelines}

Key points on antipsychotic prescribing in the updated NICE (2014) clinical guideline on psychosis and schizophrenia are summarised in Box 1.

\section{Choosing specific LAls}

Early LAIs were esterified preparations in an oil solution, administered every 1 to 6 weeks.

BOX 1 Summary of key NICE guideline points on antipsychotic medication for psychosis and schizophrenia in adults

- Do not offer antipsychotic medication to people at increased risk of developing psychosis, or to decrease the risk of, or prevent, psychosis.

- Before starting antipsychotic medication: check weight, waist circumference, pulse and blood pressure; fasting blood glucose, $\mathrm{HbA1c}$, blood lipid profile and prolactin levels; assess for any movement disorders; assess nutritional status, diet and level of physical activity; offer electrocardiogram (ECG) if specified in the drug's Summary of Product Characteristics, or physical examination has identified specific cardiovascular risk or history of cardiovascular disease, or on in-patient admission.

- Regularly throughout treatment, especially during titration, monitor and record: response to treatment, including changes in symptoms and behaviour; side-effects of treatment; emergence of movement disorders; weight, weekly for 6 weeks, at 12 weeks, at 12 months then annually (plotted on a chart); waist circumference, annually (plotted on a chart); pulse and blood pressure at 12 weeks, at 12 months, then annually; fasting blood glucose, $\mathrm{HbA} 1 \mathrm{c}$ and blood lipid levels at 12 weeks, at 1 year and then annually; adherence; and physical health.

- For initial episodes, if the person wishes to try psychological interventions alone, advise that these are more effective in conjunction with antipsychotic medication. If the person still wishes to try psychological interventions alone: offer family intervention and cognitive-behavioural therapy (CBT); agree to a time ( $\leq 1$ month) to review treatment options, including introducing antipsychotic medication; and continue regular monitoring of symptoms, distress, impairment and level of functioning, including in education, training and employment.

- For subsequent acute episodes, offer oral antipsychotic medication and psychological interventions (family intervention and individual CBT).

- The choice of antipsychotic medication should be decided between the person and healthcare professional, taking into account the views of the carer if the patient agrees.

- Provide information and discuss the likely benefits and possible side-effects of each drug, including: metabolic (including weight gain and diabetes); extrapyramidal (akathisia, dyskinesia and dystonia); cardiovascular (including prolonging OT interval); hormonal (including increasing plasma prolactin); and other (including unpleasant subjective experiences).

- Treatment with regular and 'as required' antipsychotic medication should be as follows: discuss and record side-effects that the person is most willing to tolerate; record indications, expected benefits and risks, and expected time for a change in symptoms and appearance of side-effects; at start of treatment, give a dose at lower end of the licensed range (shown in the British National Formulary or Summary of Product Characteristics) and slowly titrate upwards within the dose range given in those publications; if doses above the licensed range are used, document the reasons; record rationale for continuing changing or stopping medication, and the effects of such changes; and carry out a trial of medication at optimum dose for 4 to 6 weeks.

- Do not use a loading dose of antipsychotic medication.

- Do not initiate regular combined antipsychotic medication, except for short periods.

- Offer clozapine to people who have not responded adequately to treatment despite sequential use of at least two different antipsychotics. At least one of the drugs should be a non-clozapine second-generation antipsychotic. If response to clozapine is inadequate, consider further review, including measuring therapeutic drug levels, before adding a second antipsychotic to augment treatment with clozapine. Choose a drug that does not potentiate the common side-effects of clozapine. A trial of augmentation may need to continue for 8-10 weeks.

- Consider depot/long-acting injectable antipsychotic medication for people who would prefer this treatment after an acute episode, or where avoiding covert non-adherence (either intentional or unintentional) is a clinical priority; initially use a small test dose as set out in the British National Formulary or Summary of Product Characteristics.

(NICE 2014) 


\section{TABLE 1 LAI preparations}

\begin{tabular}{|lll|}
\hline Preparation & Dose & Comments \\
\hline Haloperidol decanoate & $50-300 \mathrm{mg}$ every 4 weeks & \\
\hline Fluphenazine decanoate & $12.5-100 \mathrm{mg}$ every 2-5 weeks & \\
\hline Flupentixol decanoate & $\begin{array}{l}50 \mathrm{mg} \text { every 2 weeks to } 300 \mathrm{mg} \\
\text { every 4 weeks }\end{array}$ & \\
\hline Zuclopenthixol decanoate & $200-500 \mathrm{mg}$ every 1-4 weeks & \\
\hline Pipotiazine palmitate & $50-100 \mathrm{mg}$ every 4 weeks & $\begin{array}{l}\text { Requires initial adjunctive oral } \\
\text { risperidone for 3-4 weeks }\end{array}$ \\
\hline Risperidone & $25-50 \mathrm{mg}$ every 2 weeks & $\begin{array}{l}\text { Monitor for post-injection } \\
\text { syndrome after each injection }\end{array}$ \\
\hline Olanzapine pamoate & $\begin{array}{l}150-300 \mathrm{mg} \text { every 2 weeks or } \\
300-405 \mathrm{mg} \text { every 4 weeks }\end{array}$ & $\begin{array}{l}\text { A loading dose at baseline and } \\
\text { day 8 can achieve peak levels } \\
\text { earlier }\end{array}$ \\
\hline Paliperidone palmitate & $25-150 \mathrm{mg}$ monthly & $\begin{array}{l}\text { Requires initial adjunctive oral } \\
\text { aripiprazole for 2-3 weeks }\end{array}$ \\
\hline Aripiprazole & 400 mg monthly &
\end{tabular}

The preparation types have broadened since the advent of risperidone LAI, which is delivered via microspheres. In recent years, newer LAI preparations have been delivered via nanoparticles in aqueous suspension for paliperidone palmitate and olanzapine pamoate, and via lyophilised powder reconstituted in sterile water for aripiprazole monohydrate (Gopalakrishna 2013). A list of available LAIs is presented in Table 1. Notwithstanding risk-benefit analyses

BOX 2 Some advantages and disadvantages of LAls over oral preparations

\section{Advantages}

- No first-pass metabolism; improved bioavailability

- More consistent delivery of antipsychotic with more stable plasma levels; minimised side-effects and reduced variations in symptom control

- Wider window of opportunity to re-engage assertively with patient if LAI refused

- Injection; plasma levels take longer to decline than with oral formulations

- Earlier detection of non-adherence, which is more overt and can be followed up quickly; potential reduction in relapse rates

- Reduced risk of accidental or intentional self-harm by overdose

\section{Disadvantages}

- Pain, erythema, swelling at the site of injection; nodule formation, particularly with oil-based injections

- Risk of damage to nerves, arteries or veins

- If side-effects occur they will be prolonged until the plasma level falls

- Patient may be allergic to the oily vehicle, so a test dose of oil-based LAls is needed

- Need to confirm efficacy and tolerability of the oral formulations of the LAls where required and practical

- Can take several weeks for plasma levels to reach steady state

- Potential logistical difficulties in administering injections to a patient who is in employment

- Possible stigma

- Patient dislike or even a phobia of needles

(After Feetam 2014: p. 12) for individual patients, LAIs have a range of advantages, but also pitfalls compared with their oral counterparts. Some of these considerations are summarised in Box 2.

\section{First-generation LAls}

For first-generation LAIs such as haloperidol, fluphenazine, flupentixol, zuclopenthixol and pipotiazine, it may take 2-3 months to achieve steady-state plasma concentrations (Taylor 2009).

The efficacy and tolerability of first-generation LAIs have been reviewed by Adams et al (2001), including their side-effect profiles in comparison with oral antipsychotics. It should be noted that Patel \& David (2005) raised the caveat that most of the randomised controlled studies included in that review were probably too short to optimise relapse prevention. This said, fluphenazine and haloperidol LAIs have higher rates of extrapyramidal sideeffects such as tardive dyskinesia, dystonias and Parkinsonism compared with the secondgeneration LAIs risperidone, paliperidone, olanzapine and aripiprazole (Gopalakrishna 2013).

\section{Second-generation LAls}

Risperidone Patients prescribed risperidone LAI require adjunctive oral risperidone for 3-4 weeks, as the biodegradable microspheres require several weeks for peak release. The medication also requires a special preparation technique and refrigeration before injection. The main tolerability problems with risperidone LAI are weight gain and hyperprolactinaemia (Gopalakrishna 2013).

Paliperidone Paliperidone LAI, based on the active metabolite of risperidone (9-hydroxyrisperidone), is available in prefilled syringes, does not require refrigeration before injection and can be administered monthly (as opposed to fortnightly for risperidone LAI); it is released as early as the first day and plasma levels peak at day 13 (an initial loading dose, followed by another at day 8 , facilitates peak levels earlier), obviating the need for oral supplementation (Gopalakrishna 2013). Paliperidone LAI is readily metabolised and thus has less potential for medication interactions than risperidone, and may be safer for patients with hepatic impairment (Álamo 2013). There are no randomised head-to-head studies comparing paliperidone LAI with risperidone LAI.

Olanzapine Olanzapine LAI reaches peak plasma levels in under a week and without the need for adjunctive oral medication. Although there is the option of monthly injections, fortnightly administration is recommended. Apart from sideeffects akin to those of oral olanzapine (namely, weight gain, sedation and hyperlipidaemia), a 
post-injection syndrome, which can occur irrespective of treatment duration, has been the main and concerning limitation with this medication. The post-injection syndrome is characterised by sedation, delirium or altered conscious state, and speech and gait disturbance (Gopalakrishna 2013). Presentations of this syndrome require monitoring and supportive care for several days, generally in intensive care, and there have been reported deaths (Kuehn 2013), possibly secondary to cardiac arrhythmia or cardiopulmonary arrest. Accordingly, patients must be monitored for 2-3 hours after each injection.

Aripiprazole Aripiprazole LAI is a lyophilised powder that requires reconstitution in sterile water. Monthly administration of aripiprazole LAI has been found to be effective at doses of 300 and $400 \mathrm{mg}$. Although peak plasma concentration occurs 5-7 days after injection, a fortnight of adjunctive oral aripiprazole has been recommended to optimise levels. Reported sideeffects include tremor, sedation, headache and increased QTc interval (Gopalakrishna 2013).

\section{LAls in clinical practice}

The available evidence indicates that LAIs are underutilised in clinical practice for people with schizophrenia (including the first episode) and possibly those with comorbid substance use disorders. Their utility and use in this context are affected by patient, clinician and service barriers that can work against best practice and evidencebased care.

Although adherence, albeit more discernible with LAIs, is a prime factor in considering these agents, the answer does not lie in a dichotomous 'oral $v$. LAI' approach to prescribing. If we are to learn from history, the therapeutic success or otherwise of LAIs essentially depends on the quality of follow-up care (Johnson 2009). Patients prescribed LAIs may relapse, even when adherent to these medications, and may also experience significant side-effects. Consequently, continued LAI use may not be appropriate for all patients, even if oral medication adherence is a significant concern. Also, differential costs of newer LAIs compared with older and cheaper agents should be balanced with individually tailored care, including considerations of tolerability.

\section{Clinician and nurse education, training and support}

In translating the evidence and clinical guidelines (such as NICE 2014) about LAI use into readily accessible, individually tailored and recoveryfocused care, concomitant support for clinician education and supervision and quality improvement processes across primary and specialist care are vital. This need is evidenced by the influence of clinicians' attitudes, which may be discordant with current guidelines, as well as the extent of awareness and vigilance about medication adherence and its marked impact on patient outcomes and relapse prevention.

Building on the essential framework of a recovery-informed approach, nurses administering LAIs have a key role in communication (in primary care and specialist settings) with other clinicians involved in patient care. This role requires engaging patients in broader discussions about their health (e.g. diet, exercise and smoking), as well as monitoring outcome measures, including tolerability (e.g. using The Liverpool University Neuroleptic Side Effect Rating Scale (LUNSERS); Day 1995). Nurses in this role, irrespective of the setting in which they are working, require initial mentoring and supervision, as well as relevant knowledge of psychopharmacology, anatomy and physiology germane to the medication. Advance choice (advance decision) conversations with the patient about the benefits and risks of treatment options such as LAIs can facilitate therapeutic engagement and care within a recovery framework (Gray 2009). The Royal College of Psychiatrists (2014) has produced a patient leaflet on LAIs, and Box 3 lists questions that may be helpful in assessing a patient's attitudes towards LAIs and oral antipsychotics.

\section{Conclusions}

The beliefs and attitudes that patients and clinicians hold about antipsychotic medication, as well as the quality of their recovery-focused relationship, are key factors in adherence. LAIs have a place in addressing non-adherence, and arguably should be part of the choices discussed

\section{BOX 3 Ascertaining the patient's attitudes towards LAls $v$. oral antipsychotics}

The following questions are helpful:

- Would you rather have medication by LAl or by tablet?

- Do you think there is a difference between taking tablets daily or having an injection every few weeks?

- How do you compare your current medication (LAl) with your previous medication?

- How do you feel about the medication you have in the form of injections compared with earlier treatment with tablets or other injections?

(Adapted from Walburn 2001) 


\section{MCQ answers}

$1 \mathrm{c} \quad 2 \mathrm{e} \quad 3 \mathrm{~d} \quad 4 \mathrm{e} \quad 5 \mathrm{~b}$ with any patient requiring long-term treatment, even early in the illness course. However, LAIs are currently underused in schizophrenia. There is a need for better education of clinicians, including improved knowledge of guidelines such as those from NICE, and for strategies to address the stigma associated with 'depot' antipsychotics and to change beliefs that are contrary to evidencebased care.

\section{References}

Achilla E, McCrone P (2013) The cost effectiveness of long-acting/ extended-release antipsychotics for the treatment of schizophrenia: a systematic review of economic evaluations. Applied Health Economics and Health Policy, 11: 95-106.

Adams CE, Fenton MKP, Quraishi S, et al (2001) Systematic meta-review of depot antipsychotic drugs for people with schizophrenia. British Journal of Psychiatry, 179: 290-9.

Addington D, Bouchard R-H, Goldberg J, et al (2005) Clinical Practice Guidelines: Treatment of Schizophrenia. Canadian Journal of Psychiatry, 50 (suppl 1): s1-56

Álamo C , López-Muñoz F (2013) The pharmacological role and clinical applications of antipsychotics' active metabolites: paliperidone versus risperidone. Clinical and Experimental Pharmacology, 3(1): 117.

Argo TR, Crismon ML, Miller AL, et al (2007) Texas Medication Algorithm Project Procedural Manual: Schizophrenia Treatment. Texas Department of State Health Services.

Barnes TRE, Shingelton-Smith A, Paton C (2009) Antipsychotic long-acting injections: prescribing practice in the UK. British Journal of Psychiatry, 195 (suppl 52): s37-42.

Chopra P, Hamilton B, Castle D, et al (2009) Implementation of the Strengths Model at an area mental health service. Australasian Psychiatry, 17: 202-6.

da Silva Freire Coutinho E, Fenton M, Quraishi SN (2009) Zuclopenthixol decanoate for schizophrenia and other serious mental illnesses. Cochrane Database of Systematic Reviews, 3: CD001164.

Day JC, Wood G, Dewey M, et al (1995) A self-rating scale for measuring neuroleptic side-effects: validation in a group of schizophrenic patients. British Journal of Psychiatry, 166: 650-3.

de Hert M, Schreurs V, Vancampfort D, et al (2009) Metabolic syndrome in people with schizophrenia: a review. World Psychiatry, 8: 15-22.

Dibonaventura M, Gabriel S, Dupclay L, et al (2012) A patient perspective of the impact of medication side effects on adherence: results of a cross-sectional nationwide survey of patients with schizophrenia. BMC Psychiatry 12: 20.

Emsley R , Oosthuizen $\mathrm{P}$, Koen L, et al (2008) Remission in patients with first-episode schizophrenia receiving assured antipsychotic medication: a study with risperidone long-acting injection. International Clinical Psychopharmacology, 23: 325-31.

Feetam C. White J (eds) (2014) Guidance on the Administration to Adults of Oil-based Depot and Other Long-Acting Intramuscular Antipsychotic Injections (4th edn). University of Hull (http://www2.hull.ac.uk/fhsc/pdf/ SOP_Final_2014_PDF.pdf).

Gopalakrishna G, Aggarwal A, Lauriello J (2013) Long-acting aripiprazole: how might if fit into our tool box? Clinical Schizophrenia \& Related Psychoses, 7: 87-92.

Gray R, Spilling R, Burgess D, et al (2009) Antipsychotic long-acting injections in clinical practice: medication management and patient choice. British Journal of Psychiatry, 195 (suppl 52): s51-6.

Green Al, Drake RE, Brunette MF, et al (2007) Schizophrenia and cooccurring substance use. American Journal of Psychiatry, 164: 402-8.

Green A (2012) Long-acting injectable antipsychotic medications in patients with comorbid schizophrenia and substance use disorders. Journal of Dual Diagnosis, 8: 62-3.
Haddad P, Lambert T, Lauriello J (2011) The role of antipsychotic longacting injections in current practice. In Antipsychotic Long-Acting Injections (eds P Haddad, T Lambert, J Lauriello): 241-60. Oxford University Press.

Haro JM, Suarez D, Novick D, et al (2007) Three-year antipsychotic effectiveness in the outpatient care of schizophrenia: observational versus randomized studies results. European Neuropsychopharmacology, 17: 235-44.

Harrow M, Jobe TH (2007) Factors involved in outcome and recovery in schizophrenia patients not on antipsychotic medications: a 15-year multifollow-up study. Journal of Nervous and Mental Disease, 195: 406-14

Heres S, Schmitz FS, Leucht S, et al (2007) The attitude of patients towards antipsychotic depot treatment. International Clinical Psychopharmacology, 22: 275-82.

Jaeger M, Rossler M (2010) Attitudes towards long-acting antipsychotics: a survey of patients, relatives and psychiatrists. Psychiatry Research 175: 58-62.

Johnson DAW (2009) Historical perspective on antipsychotic long-acting injections. British Journal of Psychiatry, 195 (suppl 52): s7-12.

Kane JM, Garcia-Ribera C (2009) Clinical guideline recommendations for antipsychotic long-acting injections. British Journal of Psychiatry, 195 (suppl 52): s63-7.

Kirson NY, Weiden PJ, Yermakov S, et al (2013) Efficacy and effectiveness of depot versus oral antipsychotics in schizophrenia: synthesising results across different research designs. Journal of Clinical Psychiatry, 74: 568-75.

Koola MM, Wehring HJ, Kelly DL (2012) The potential role of long-acting injectable antipsychotics in people with schizophrenia and comorbid substance use. Journal of Dual Diagnosis, 8: 50-61.

Kreyenbuhl J, Buchanan RW, Dickerson FB, et al (2010) The Schizophrenia Patient Outcomes Research Team (PORT): updated treatment recommendations 2009. Schizophrenia Bulletin, 36: 94-103.

Kuehn BM (2013) FDA investigates deaths related to use of olanzapine. JAMA, 310: 361

Lehman AF, Lieberman JA, Dixon LB, et al (2010) Practice Guideline for the Treatment of Patients with Schizophrenia (2nd edn). American Psychiatric Association.

Leucht C, Heres S, Kane JM, et al (2011) Oral versus depot antipsychotic drugs for schizophrenia: a critical systematic review and meta-analysis of randomised controlled long term trials. Schizophrenia Research, 127: 83-92.

McCabe R, Bullenkamp J, Hansson L, et al (2012) The therapeutic relationship and adherence to antipsychotic medication in schizophrenia. Plos One, 7 (4): e36080.

McEvoy JP, Byerly M, Hamer RM, et al (2014) Effectiveness of paliperidone palmitate vs haloperidol decanoate for maintenance treatment of schizophrenia: a randomized clinical trial. JAMA, 311: 1978-87.

National Institute for Health and Care Excellence (2014) Psychosis and Schizophrenia in Adults: Treatment and Management (NICE Clinical Guideline 178). NICE.

O'Donoghue B, Lyne J, Renwick L, et al (2012) A descriptive study of 'noncases' and referral rates to an early intervention for psychosis service. Schizophrenia Research, 6: 276-82.

Offord S, Wong B, Mirski D, et al (2013) Healthcare resource usage of schizophrenia patients initiating long-acting injectable antipsychotics vs oral. Journal of Medical Economics, 16: 231-9.

Olfson M, Marcus SC, Ascher-Svanum H (2007) Treatment of schizophrenia with long-acting fluphenazine, haloperidol, or risperidone. Schizophrenia Bulletin, 33: 1379-87.

Patel MX, David AS (2005) Why aren't depot antipsychotics prescribed more often and what can be done about it? Advances in Psychiatric Treatment, 11: 203-11.

Patel MX, Taylor M, David AS (2009) Antipsychotic long-acting injections: mind the gap. British Journal of Psychiatry, 195 (suppl 52): s1-4. 
Patel MX, Haddad PM, Chaudhry IB, et al (2010) Psychiatrists' use, knowledge and attitudes to first- and second-generation antipsychotic long acting injections: comparisons over 5 years. Journal of Psychopharmacology, 24: 1473-82

Rosenheck RA, Krystal JH, Lew R, et al (2011) Long-acting risperidone and oral antipsychotics in unstable schizophrenia. New England Journal of Medicine, 364: 842-51.

Royal College of Psychiatrists (2014) Depot medication. Royal College of Psychiatrists (http://www.rcpsych.ac.uk/healthadvice/ treatmentswellbeing/depotmedication.aspx).

Rubio G, Martinez I, Ponce G, et al (2006) Long-acting injectable risperidone compared with zuclopenthixol in the treatment of schizophrenia with substance abuse comorbidity. Canadian Journal of Psychiatry, 51: 531-8.

Stanhope V, Marcus S, Solomon P (2009) The impact of coercion on services from the perspective of mental health care consumers with cooccurring disorders. Psychiatric Services, 60: 183-8.

Staring ABP, Van der Gaag M, Koopmans GT, et al (2010) Treatment adherence therapy in people with psychotic disorders: randomised controlled trial. British Journal of Psychiatry, 197: 448-55.

Taylor D (2009) Psychopharmacology and adverse effects of antipsychotic long-acting injections: a review. British Journal of Psychiatry, 195 (suppl 52): $s 13-9$

Tiihonen J, Wahlbeck K, Lonnqvist J, et al (2006) Effectiveness of antipsychotic treatments in a nationwide cohort of 2230 patients in community care after first hospitalisation due to schizophrenia and schizoaffective disorder: observational follow-up study. BMJ 333: 224.
Tiihonen J, Haukka J, Taylor M, et al (2011) A nationwide cohort study of oral and depot antipsychotics after first hospitalization for schizophrenia. American Journal of Psychiatry, 168: 603-9.

Vázquez Vázquez JM, González-Rodríquez A, Sanz Asin P, et al (2012) EPA-0354 - Efficacy and tolerability of long-acting injectable paliperidone palmitate in the treatment of dual psychosis [Abstract]. European Psychiatry, 29 (suppl 1): 1

Velligan DI, Wang M, Diamond P, et al (2007) Relationships among subjective and objective measures of adherence to oral antipsychotic medications. Psychiatric Services, 58: 1187-92.

Waddell L, Taylor M (2009) Attitudes of patients and mental health staff to antipsychotic long-acting injections: systematic review. British Journal of Psychiatry, 195 (suppl 52): s43-50.

Walburn J, Gray R, Gournay K, et al (2001) Systematic review of patient and nurse attitudes to depot antipsychotic medication. British Journal of Psychiatry, 179: 300-7.

West JC, Marcus SC, Wilk J, et al (2008) Use of depot antipsychotic medications for medication nonadherence in schizophrenia. Schizophrenia Bulletin, 34: 995-1001.

Zhornitsky S, Stip E (2012) Oral versus long-acting injectable antipsychotics in the treatment of schizophrenia and special populations at risk for treatment nonadherence: a systematic review. Schizophrenia Research and Treatment, 2012: ID 407171.

Zhu B, Ascher-Svanum H, Shi L, et al (2008) Time to discontinuation of depot and oral first generation antipsychotics in the usual care of schizophrenia. Psychiatric Services, 59: 315-7.

\section{MCOs}

Select the single best option for each question stem

1 Long-acting injectable antipsychotics (LAIs):

a are generally better tolerated than oral antipsychotics

b prevent relapse in more than $95 \%$ of patients with schizophrenia

c are less subject to covert non-adherence than oral antipsychotics

$\mathrm{d}$ are administered at least every 4 weeks

e are more efficacious than similar oral antipsychotics.

\section{Treatment adherence for LAls:}

$\mathrm{a}$ is to a small degree dependent on the quality of the patient's relationship with the clinicians

$\mathrm{b}$ is worse than for oral antipsychotic medications

c is occasionally related to the patient's personal opinion regarding their susceptibility to relapse $\mathrm{d}$ is generally predicted by a patient's verbal report of their adherence behaviour

$\mathrm{e}$ is significantly influenced by the side-effect profile.

3 Reasons for LAI underutilisation do not include:

a prescriber knowledge regarding these medications

b family or carer views

c patients' concerns about pain and side-effects

d sufficient information provided to the majority of patients

e the cost of medications.

\section{Advantages of LAls compared with oral} antipsychotics include:

a easier early detection of relapse

b better cardiometabolic tolerability in a proportion of patients c less need to focus on a recovery-based approach to care

d markedly reduced hospital readmission rates

e reduced risk of self-harm with antipsychotic medication.

5 Regarding the NICE clinical guidelines for psychosis and schizophrenia in adults:

a physical health evaluation is sometimes required before initiation of LAls

b LAls should be considered when avoiding covert non-adherence is a clinical priority

c LAls should be avoided in people after a first episode

d clinicians' views are more important than patients' views about LAls

e LAls delay the need for clozapine. 